\title{
Educação para a Saúde e Prevenção na Consulta de Medicina Geral e Familiar (I)
}

Alberto Pinto Hespanhol,* Luciana Couto, ${ }^{* *}$ Carlos Martins, ${ }^{* * *}$ Manuel Viana, ${ }^{* * * *}$

\section{RESUMO}

Neste artigo, o termo medicina preventiva deve ser entendido como sinónimo de promoção da saúde, a qual, segundo a Carta de Ottawa (OMS, 1986), consiste no processo que visa criar condições para que as pessoas aumentem a sua capacidade de controlar os factores determinantes da saúde, no sentido de a melhorar. A educação para a saúde e a prevenção são componentes da promoção da saúde.

Baseado nos princípios da medicina preventiva, discutem-se alguns aspectos relacionados com a consulta de Medicina Geral e Familiar: medicina baseada na evidência e medicina preventiva.

Palavras-Chave: Medicina Preventiva; Promoção da Saúde; Educação para a Saúde; Prevenção; Medicina Baseada na Evidência.

\section{ENQUADRAMENTO CONCEPTUAL}

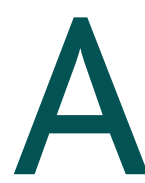

Wonca Europa, com o apoio e a colaboração da OMS Europa, refere, em 2002, na sua Declaração sobre a nova Definição Europeia de Medicina Geral e Familiar que «...A Medicina Geral e Familiar (Clínica Geral/Medicina Familiar) é uma disciplina académica e científica, com os seus próprios conteúdos educacionais, investigação, base de evidência e actividade clínica; é uma especialidade clínica orientada para os Cuidados de Saúde Primários...". ${ }^{1}$

É neste contexto que se desenvolve, em Medicina Geral e Familiar, a Promoção da Saúde dos principais problemas que afectam a saúde dos portugueses, ${ }^{2}$ definidos em função dos dados de prevalência comunitária. ${ }^{3-9}$ Esse objectivo diz, portanto, respeito aos esforços para melhorar a dimensão positiva da saúde dos Portugueses e prevenir a sua dimensão negativa, através

*Professor Convidado Associado com Agregação/Assistente Graduado de Clínica Geral. Departamento Clínica Geral da Faculdade de Medicina da Universidade do Porto. Centro Saúde São João da Faculdade de Medicina da Universidade do Porto **Professora Convidada Auxiliar/Assistente Graduada de Clínica Geral. Departamento Clínica Geral da Faculdade de Medicina da Universidade do Porto. Unidade Saúde Familiar das Camélias

***Assistente de Clínica Geral.

Departamento Clínica Geral da Faculdade de Medicina da Universidade do Porto. Centro Saúde São João da Faculdade de Medicina da Universidade do Porto ****Assistente Graduado de Clínica Geral.

Departamento Clínica Geral da Faculdade de Medicina da Universidade do Porto. Centro Saúde São João da Faculdade de Medicina da Universidade do Porto das esferas sobrepostas da Educação para a Saúde, Prevenção da doença e Protecção da saúde. ${ }^{10}$

Essa acção em Promoção da Saúde ${ }^{2}$ implica o desenvolvimento de diferentes actividades, que podem ser sistematizadas em três vertentes de acção inter-relacionadas e complementares:

- Educação para a saúde - processo que utiliza a comunicação pedagógica no sentido de facilitar a aprendizagem da saúde;

- Prevenção da doença - conjunto de medidas que visam evitar, detectar e tratar precocemente doenças específicas e eventuais sequelas;

- Protecção da saúde - conjunto de medidas destinadas ao controlo de factores de risco de natureza ambiental e à preservação dos recursos naturais. ${ }^{10}$

É, também, nesta direcção, que vai o Modelo de Promoção da Saúde de Tannahill, segundo o qual a Prevenção da doença consiste na redução do risco da ocorrência da dimensão negativa da saúde e apresenta áreas de sobreposição, confluência e interligação com a educação para a saúde e com a protecção da saúde. ${ }^{10}$

Em artigo por nós recentemente publicado ${ }^{11} \mathrm{e}$ a propósito do enquadramento conceptual da Medicina Preventiva, citamos a classificação de Tones, ${ }^{10}$ que hierarquiza a Educação para a Saúde em três estratos, à semelhança da Prevenção da doença, e entre os quais es- 
tabelece conexões:

- a prevenção primária pretende reduzir a incidência de doença na população, enquanto a educação para a saúde primária visa a promoção do comportamento que proporcione bem-estar ou evite a doença;

- a prevenção secundária pretende reduzir a prevalência de doença na população, enquanto a educação para a saúde secundária visa a promoção de realização de rastreios, equacionados para a detecção de doença em indivíduos assintomáticos assim como a utilização adequada dos Serviços de Saúde;

- a prevenção terciária pretende reduzir a incapacidade provocada pela doença já estabelecida, enquanto a educação para a saúde terciária visa a promoção da adesão à terapêutica, assim como a utilização dos serviços de reabilitação. ${ }^{10}$

A Educação para a Saúde, segundo a Organização Mundial de Saúde (1969), «... diz respeito a todas as experiências do indivíduo, grupo ou comunidade que influenciam crenças, atitudes ou comportamentos referentes à saúde, assim como aos modos e aos esforços de produzir a mudança, quando isso énecessário para uma óptima saúde». ${ }^{12,13}$

O conceito de Educação para a Saúde engloba, pois, dois aspectos distintos, embora complementares - a informação dos utentes sobre a saúde e a mudança de comportamento perante a saúde.

Segundo este conceito, a Educação para a Saúdenão é só saber mais, mas, também, comportar-se de modo diferente. Contudo não podemos entender que a única Educação para a Saúde válida é aquela que pode ser avaliada em termos de mudança comportamental, muito embora seja também de esperar que ocorra alguma mudança de comportamento.

Na literatura médica têm vindo a ser identificados uma série de factores que aumentam a eficácia da $E d u$ cação para a Saúde prestada pelos Clínicos Gerais / Médicos de Família (CG/MF), ou seja, uma melhoria da adesão à terapêutica prescrita ou a conselhos fornecidos nas áreas preventivas pelos médicos. São exemplo destes factores:

- a satisfação do utente com o seu médico;

- a continuidade de prestação de cuidados médicos;

- o pouco tempo de espera pela consulta;

- a organização adequada da unidade de saúde;

- a vigilância médica contínua da terapêutica;
- o encorajamento à auto-vigilância do utente e o regime terapêutico simples; ${ }^{12}$

- o sentimento de confiança no CG/MF por parte do utente; ${ }^{14}$

- o envolvimento do utente no processo de tomada de decisão durante a consulta; ${ }^{15}$

- a identificação dos benefícios e dos custos; ${ }^{16}$

- a implementação de estratégias que visam ajudar os utentes a recordar conselhos preventivos previamente administrados. ${ }^{17}$

A educação para a saúde e a prevenção da doença podem ser analisadas por três ângulos diferentes, que se pretende que sejam compatíveis: a perspectiva dos utentes, a perspectiva da relação médico/utente e a perspectiva dos médicos.

A perspectiva dos utentes acerca das capacidades interpessoais dos médicos está fortemente relacionada com a confiança, mas também com a empatia, a segurança e o apoio evidenciados no relacionamento terapêutico e com a existência de médicos que oiçam as suas preocupações e as suas preferências e lhes prestem uma informação sincera sobre a sua situação clínica e sobre as suas opções de tratamento. ${ }^{18}$ Os atributos que os utentes mais frequentemente associam à confiança são a honestidade, a franqueza, a sensibilidade e o ter em mente os melhores interesses do doente. ${ }^{18}$

Na perspectiva da relação médico/utente, podem surgir problemas, quando se confrontam as expectativas cada vez maiores, expressas pelos utentes, no sentido de serem informados acerca da sua saúde e dos seus problemas, com a relutância dos CG/MF em aceder a essas solicitações.

Na perspectiva dos médicos, múltiplas razões podem justificar esta sua relutância: o cepticismo com que encaram a eficácia da educação para a saúde, o receio de provocar ansiedade entre os seus utentes ao realizá-la ou a dificuldade que sentem em executá-la, por falta de tempo. ${ }^{12,13}$

A educação para a saúde e a prevenção da doença, ao nível da especialidade de Medicina Geral e Familiar, podem ser feitas segundo duas abordagens ou estratégias diferentes: uma abordagem comunitária ou estratégia populacional, menos personalizada, dirigida às populações, mais institucional, ao nível dos Centros de Saúde e outras organizações comunitárias e uma abordagem familiar ou estratégia individual, mais persona- 
lizada, dirigida aos indivíduos e às suas famílias, ao nível da relação médico/utente, ou seja, a nível da consulta. $^{11,19}$

Embora a generalidade dos autores refira que a consulta é uma aptidão comum às diferentes formas de exercício da profissão médica, em Medicina Geral e Familiar esse encontro singular entre uma pessoa necessitada de auxílio e um médico que lho pode dar, é, seguramente, o seu instrumento privilegiado de trabalho e tem vindo a ser considerado o veículo mais eficaz e mais credível de Educação para a Saúde e de Prevenção da Doença..$^{20-22}$

Para que a consulta seja um meio adequado da relação médico/utente numa perspectiva global, é preciso que os médicos estejam conscientes dos factores essenciais da comunicação frente a frente, assim como das diferentes modalidades de comunicação na consulta, que constituem a mensagem do utente para o médico e a retro-informação deste para o utente. Por outro lado, também é importante que saibam que, para que a consulta seja um veículo adequado de transmissão de informações, é, também, necessário, que os médicos dominem e executem determinadas técnicas de administração de instruções e de conselhos aos utentes. ${ }^{21,23}$

Não sendo possível abordar exaustivamente todos os temas relativos à Educação para a Saúde e à Prevenção na Consulta de Medicina Geral e Familiar, seleccionamos alguns aspectos que nos parecem, pela sua importância, merecer uma reflexão especial, à luz da medicina preventiva. No presente artigo, Educação para a Saúde e Prevenção na Consulta de Medicina Geral e Familiar (I), é abordada a Medicina Baseada na Evidência e a Medicina Preventiva e no artigo seguinte, Educação para a Saúde e Prevenção na Consulta de Medicina Geral e Familiar (II), são abordados os Rastreios a nível dos ciclos vitais individual e familiar e os Rastreios oncológicos.

\section{MEDICINA BASEADA NA EVIDÊNCIA E A MEDICINA PREVENTIVA}

Numa perspectiva histórica, o desenvolvimento da Medicina Preventiva e a aplicação de actividades preventivas teve uma relação estreita com o conceito de exame periódico de saúde. Segundo Last JM, o exame periódico de saúde corresponde a uma avaliação do estado de saúde, conduzida em intervalos pré-determinados, por exemplo, anualmente, que segue um determinado protocolo, em que se aplica um conjunto estruturado de questões e um conjunto pré-determinado de exames ou testes laboratoriais. ${ }^{24}$ Uma das primeiras recomendações para a realização de um exame periódico de saúde data de 1922, ano em que a American Medical Association propôs a realização de um exame médico anual a todos os adultos, que incluía uma bateria de testes de diagnóstico. ${ }^{25}$

Durante o século XX, as alterações da sociedade, a evolução da medicina, a generalização das vacinas e do uso dos antibióticos, a melhoria das condições de higiene e sanitárias e a generalização da rede de frio foram alguns dos factores que contribuíram para a necessidade de um ajustamento no equilíbrio «necessidades versus oferta» dos serviços de saúde. No princípio do século XX, as principais causas de mortalidade relacionavam-se com doenças infecto-contagiosas. No final do século XX, as principais causas de mortalidade relacionavam-se com doenças crónicas. A emergência das doenças crónicas registada na segunda metade do século XX e o reconhecimento de que uma parte significativa das mesmas pode ser prevenível, associados ao boom tecnológico na área dos exames complementares de diagnóstico (análises, exames de imagem, etc.) contribuiu para a atracção crescente pela Medicina Preventiva.

Nos anos 70, Frame PS e Carlson SJ efectuaram uma revisão crítica da eficácia das actividades preventivas aplicadas por rotina no exame periódico de saúde e sugeriram que a selecção de actividades preventivas aplicadas no exame periódico de saúde de adultos assintomáticos fosse efectuada de acordo com a eficácia demonstrada e ajustadas, especificamente, para a idade e sexo dos adultos. ${ }^{25,26}$ Esta análise crítica contribuiu para o debate sobre a eficácia de alguns testes que estavam a ser aplicados, de uma forma generalizada, com intenção preventiva. ${ }^{27}$ É neste contexto que surge, em 1976, a Canadian Task Force on the Periodic Health Examination, actualmente denominada Canadian Task Force on Preventive Health Care (CTFPHC), com o objectivo de avaliar a evidência científica e emitir recomendações sobre as actividades preventivas que deveriam ser implementadas no exame periódico de saúde efectuado a adultos assintomáticos..$^{28,29}$ Com objectivos semelhantes, surgiu, em 1984, a United States Preven- 
tive Services Task Force (USPSTF). O aparecimento da CTFPHC e da USPSTF coincidiu com o surgimento da Medicina Baseada na Evidência (MBE), o que permitiu a adopção de métodos de revisão científica, que conduziram à publicação de recomendações de actividades preventivas, de acordo com uma escala baseada em níveis e graus de evidência. ${ }^{30-32}$ Desde então, as recomendações têm sido actualizadas, adoptadas e adaptadas por vários grupos de trabalho a nível internacional. ${ }^{22,33-36}$ Desta forma, os clínicos obtiveram um conjunto de ferramentas práticas que os orienta, com base na melhor evidência científica, na selecção das actividades preventivas que devem aplicar aos seus pacientes, o que constituiu um avanço significativo na qualidade do desempenho da Medicina Preventiva.

Contudo, tal como a MBE, a implementação das recomendações de actividades preventivas implica, sempre, a melhor integração de três componentes fundamentais: a evidência resultante da melhor investigação científica, a perícia do clínico e os valores pessoais do paciente, de acordo com as circunstâncias em que o mesmo se encontra. ${ }^{37}$ A questão que se coloca é a de saber como, na actualidade, poderá ser aplicada a MBE? A prática integral da MBE poderá, de acordo com Straus et al., compreender cinco passos: ${ }^{37}$

- Passo 1-Converter a necessidade de informação em questão.

- Passo 2 - Pesquisar a melhor evidência com a qual se responderá à questão.

- Passo 3 - Analisar criticamente a evidência encontrada, no que respeita à sua validade, impacto e aplicabilidade.

- Passo 4 - Integrar o resultado com a perícia clínica e com a especificidade biológica, de valores e circunstâncias do paciente.

- Passo 5 - Avaliar a efectividade e a eficiência na execução dos passos 1 a 4 .

Os mesmos autores consideram que poderão ser consideradas três formas de integrar a melhor evidência na prática clínica:

a) Modo «fazer» («doing mode»): os clínicos aplicam, pelo menos, os passos 1 a 4 .

b) Modo «usar»(«using mode»): a pesquisa é restrita a fontes de evidência já submetidas a uma análise prévia por terceiros (p. ex. resumos de evidência), ultrapassando, desta forma, o passo 3.

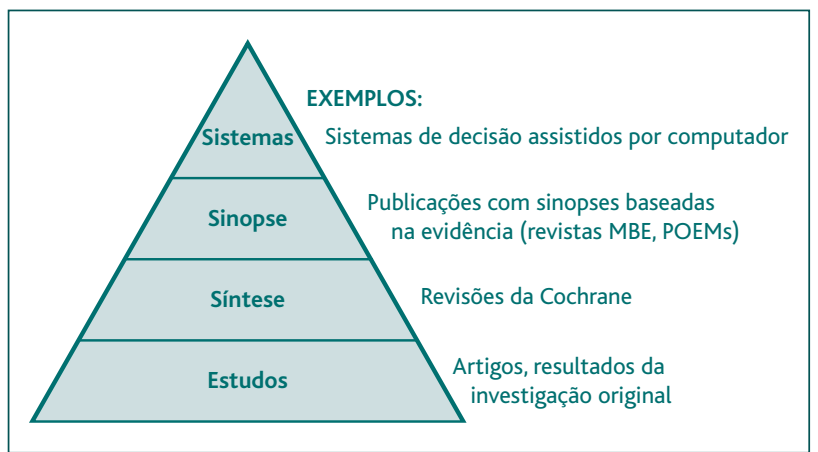

Figura 1. Organização da informação/evidência proveniente da investigação.

Traduzido e adaptado de Straus SE, Richardson WS, Glasziou P, Haynes RB. Evidence-based medicine: how to practice and teach EBM. 3rd ed. Oxford: Elsevier; 2005.

c) Modo «replicar» («replicating mode»): os clínicos seguem as decisões de líderes de opinião respeitados, não aplicando os passos 2 e 3.

Todos estes modos envolvem a integração da evidência (qualquer que seja a fonte) com a especificidade biológica, de valores e circunstâncias do paciente, ou seja, o passo 4, mas variam nos restantes passos.

O modo «usar» é o modo mais acessível e mais praticado ${ }^{38} \mathrm{~A}$ informação à disposição dos clínicos é imensa e torna-se difícil manter uma actualização constante sobre todas questões que vão surgindo na prática clínica. A Figura 1 ilustra uma possível organização da informação.

Regressando à Medicina Preventiva, é fácil reconhecer que, neste âmbito, os médicos têm já, à sua disposição, ferramentas que lhes permitem assumir o modo «usar» na integração da melhor evidência na sua prática clínica. As recomendações de actividades preventivas publicadas, regularmente, pela USPSTF e pelo Colégio Australiano de Médicos de Família são resumos de evidência de excelente qualidade, que correspondem às sinopses da pirâmide informação. ${ }^{22,33}$ Entretanto, a USPSTF lançou, recentemente, um selector electrónico de actividades preventivas (ePSS - http://epss.ahrq.gov) disponível na internet e, também, para computador de bolso, que corresponde a um sistema de suporte de decisão assistido por computador. Um outro sistema de suporte de decisão assistido por computador para os clínicos que desejem completar a sua informação, embora não exclusivamente do âmbito preventivo, é o 


\section{UpToDate $^{\circledast}$ (http://www.uptodate.com).}

Estas ferramentas podem contribuir para a eficiência da Medicina Preventiva, contudo, não deverá, nunca, ser esquecido o adequado desempenho do passo 4 , nem descurada a observância dos princípios éticos, que, no âmbito da Medicina Preventiva, tem sido alvo de intensa reflexão. ${ }^{11,39}$

\section{REFERÊNCIAS BIBLIOGRÁFICAS}

1. Evans P, Ram P, Svab I, Heyrman J, Crebolder H, Gay B, et al. A Definição Europeia de Medicina Geral e Familiar - versão Portuguesa ( traduzida por Rui Pombal). Barcelona, Espanha:Wonca Europa; 2002. Disponível em: http://www.apmcg.pt/files/54/documentos/2007060115 471793311.pdf [acedido em 30/11/08].

2. WHO. Ottawa Charter for Health Promotion. An international conference on health promotion: The move towards a new public health; 1986 Nov 17-21; Ottawa. Ottawa:WHO; 1987.

3. INSA. Inquérito Nacional de Saúde 1998/1999. Lisboa: INSA; 2001.

4. Cabral M, Silva $P$, Mendes $H$. Saúde e Doença em Portugal - Inquérito aos comportamentos e atitudes da população portuguesa perante o sistema nacional de saúde. Lisboa: Imprensa de Ciências Sociais da Universidade de Lisboa; 2002.

5. Barrros PP. Estilos de vida e estado de saúde: uma estimativa da função de produção de saúde. Rev Port Saúde Pública 2003; vol. temático 3: 7-17.

6. Portugal. Ministério da Saúde. Direcção-Geral da Saúde. Risco de morrer em Portugal, 2001. Lisboa. Direcção-Geral da Saúde, 2003.

7. Portugal. Ministério da Saúde. Direcção-Geral da Saúde. Plano Nacional de Saúde (2004-2010): volume 1 - Prioridades. Lisboa. Direcção-Geral da Saúde, 2004.

8. OECD. OECD Health Data 2006. A Comparative Analysis of 30 Countries. Credes; 2006.

9. INSA, INE. Inquérito Nacional de Saúde 2005/2006. Lisboa: INSA/INE; 2006.

10. Downie RS, Fyfe C, Tannahill. Health Promotion, Models and Values. Oxford: Oxford University Press; 1990.

11. Hespanhol A, Couto L, Martins C. A Medicina Preventiva. Rev Port Clin Geral; 2008 Jan-Fev; 24 (1): 49-64.

12. Smail S. Patient education in general practice. In: Gray $\mathrm{M}$, Fowler $\mathrm{GH}$. Preventive Medicine in General Practice. Oxford: Oxford University Press; 1983. p. 56-69.

13. Hespanhol A. Problemas éticos em Medicina Geral e Familiar. Rev Port Clin Geral 2003 Jul-Ago; 19 (4): 389-93.

14. Trachtenberg F, Dugan E, Hall M. How patients' trust relates to their involvement in medical care. J Fam Pract 2005 Apr; 54 (4): 344-52.

15. Rao J,Weinberger M, Kroenke K.Visit-specific expectations and patient centred outcomes: a literature review. Arch Fam Med 2000 Nov-Dec; 9 (10): 1148-55.

16. Schauffler $H$, Rodriguez T, Milstein A. Health education and patient satisfaction. J Fam Pract 1996 Jan; 42 (1): 62-8.

17. Ley P. Patients' understanding and recall in clinical communication failure. In: Pendleton D, Hasler J, editors. Doctor-patient communication. London: Academic Press; 1983.
18. Coulter A. Patients' views of the good doctor. BMJ 2002 Sep 28; 325 (7366): 668-9.

19. Hespanhol A. A família e os estilos de vida (Parte I). Familiarmente 1999; $2(1): 5-8$.

20. Pinto AS. Especificidade da clínica geral. Rev Port Clin Geral 1989 Nov-Dez; 6 (6): 184-5.

21. Nunes JM. Comunicação em contexto clínico. Lisboa: Bayer Health Care; 2007.

22. The Royal Australian College of General Practitioners. Guidelines for preventive activities in general practice. 6 th ed. Melbourne: Royal Australian College of General Practitioners; 2005.

23. Pendleton D, Schofield T, Tate P, Havelock P. A consulta: uma abordagem à aprendizagem e ensino. Porto: Departamento de Clínica Geral da Faculdade de Medicina do Porto; 1993.

24. Last JM. A Dictionnary of Epidemiology. Oxford: Oxford University Press; 2001.

25. Beck LH. Periodic health examination and screening tests in adults. Hosp Pract 1999 Dec; 34 (12): 117-8.

26. Frame PS, Carlson SJ. A critical review of periodic health screning using specific screening criteria. Parts 1-4. J Fam Pract 1975; 2: 29-36, 2: $123-$ 9, 2: 189-94, 2: 283-9.

27. Breslow L, Somers AR. The lifetime health-monitoring program: a practical approach to preventive medicine. N Engl J Med 1977 Mar 17; 296 (11): 601-8.

28. Canadian Task Force on the Periodic Health Examination. The Periodic Health Examination. Can Med Assoc J 1979 Nov 3; 121 (9): 1193-254.

29. Canadian Task Force on Preventive Health Care. CTFPHC History/Methodology. Disponível em URL: http://www.ctfphc.org/ctfphc\&methods.htm [acedido em 07/05/2008].

30. Evidence-Based Medicine Working Group. Evidence-based medicine: a new approach to teaching the practice of medicine. JAMA $1992 \mathrm{Nov}$ 4; 268 (17): 2420-5.

31. United States Preventive Services Task Force. Guide to Clinical Preventive Services. 2nd ed. Washington, DC: U.S. Department of Health and Human Services; 1996.

32. Canadian Task Force on the Periodic Health Examination. Canadian Guide to Clinical Preventive Health Care. Ottawa: Health Canada; 1994.

33. United States Preventive Services Task Force. The Guide to Clinical Preventive Services, 2007. Washington, DC:AHRQ; 2007.

34. Pérula de Torres LA, Alonso Arias A, Bauzà Nicolai K, Estévez JC, Iglesias Rodal M, Martín Carrillo $P$, et al. Opiniones de los profesionales sanitarios sobre la influencia del Programa de Actividades Preventivas y de Promoción de la Salud (PAPPS) en atención primaria. Aten Primaria 2007 Nov; 39 Supl 3: 5-14.

35. PAPPS. Recomendaciones PAPPS. Barcelona: Semfyc Ediciones; 2003.

36. PAPPS. Actividades preventivas en la infancia y adolescencia. Barcelona: Semfyc Ediciones; 2004.

37. Straus SE, Richardson WS, Glasziou P, Haynes RB. Evidence-based medicine: how to practice and teach EBM. 3rd ed. Oxford: Elsevier; 2005.

38. McColl A, Smith H, White P, Field J. General practicioners' perceptions of the route to evidence-based medicine: a questionnaire survey. BMJ 1998 Jan 31; 316 (7128): 361-5.

39. Gérvas J, Starfield B, Heath I. Is clinical prevention better than cure? Lancet 2008 Dec 6; 372 (9654): 1997-9. 


\section{ENDEREÇO PARA CORRESPONDÊNCIA}

Alberto Augusto Oliveira Pinto Hespanhol

Centro Saúde São João - Miguel Bombarda, 234-4050 Porto

Tel. 223395370 - Fax. 223395371

E-mail: direccao@cssjoao.min-saude.pt

\section{ABSTRACT}

In this article the term preventive medicine should be understood as a synonym of health promotion, which according to Ottawa Charter (OMS, 1986) consists of the process that aims to create conditions so that people increase its capacity to control the determinative factors of health, towards its improvement. Health education and prevention are components of health promotion. Based on the principles of preventive medicine, we discuss some aspects related to the Consultation of Family Medicine: evidence based medicine and preventive medicine.

Keywords: Preventive Medicine; Health Promotion; Health Education; Prevention; Evidence Based Medicine. 